\title{
A Field-Deployable Diagnostic Assay for the Visual Detection of Misfolded Prions
}

\section{Author Information}

Peter R. Christenson ${ }^{1,2}$, Manci Li ${ }^{2,3}$, Gage Rowden ${ }^{2,3}$, Marc Schwabenlander ${ }^{2,3}$, Tiffany M. Wolf ${ }^{2,4}$, SangHyun $\mathrm{Oh}^{1,2}$, Peter A. Larsen ${ }^{2,3^{*}}$

Affiliations

${ }^{1}$ Department of Electrical and Computer Engineering, University of Minnesota, Minneapolis, MN 55455, USA

${ }^{2}$ Minnesota Center for Prion Research and Outreach, University of Minnesota, St. Paul, MN 55108, USA

${ }^{3}$ Department of Veterinary and Biomedical Sciences, University of Minnesota, St. Paul, MN 55108, USA

${ }^{4}$ Department of Veterinary and Population Medicine, University of Minnesota, St. Paul, MN 55108, USA

\section{Contributions}

PRC and ML conceived the study. PRC, ML, and GR performed molecular experiments. PRC, ML, MS, SHO, and PAL assisted with experimental design and interpreted the results. PRC, ML, MS, TMW, and PAL conducted field-based testing of the diagnostics presented herein. PRC and ML performed statistical analyses. SHO and PAL oversaw the research. All authors wrote and contributed to the final manuscript.

\section{Corresponding author}

Peter A. Larsen

Email: plarsen@umn.edu 


\begin{abstract}
Diagnostic tools for the detection of protein-misfolding diseases (i.e., proteopathies) are limited. Gold nanoparticles (AuNPs) facilitate sensitive diagnostic techniques via visual color change for the detection of a variety of targets. In parallel, recently developed quaking-induced conversion (QuIC) assays leverage protein-amplification and fluorescent signaling for the accurate detection of misfolded proteins. Here, we combine AuNP and QuIC technologies for the visual detection of amplified misfolded prion proteins from tissues of wild white-tailed deer infected with chronic wasting disease (CWD), a prion disease of cervids. Our newly developed assay, MN-QuIC ${ }^{\mathrm{TM}}$, enables both naked-eye and light-absorbance measurements for detection of misfolded prions. MN-QuIC ${ }^{\mathrm{TM}}$ leverages basic laboratory equipment that is cost-effective and portable, thus facilitating real-time prion diagnostics across a variety of settings. To test the portability of our assay, we deployed to a rural field-station in southeastern Minnesota and tested for CWD on site. We successfully demonstrated that MN-QuIC ${ }^{\mathrm{TM}}$ is functional in a non-traditional laboratory setting by performing a blinded analysis in the field and correctly identifying all CWD positive and CWD not detected deer at the field site in less than 24 hours, thus documenting the portability of the assay. Additionally, we show that electrostatic forces help govern the AuNP/prion interactions.

Importantly, all of our white-tailed deer $(\mathrm{n}=37)$ were independently tested using ELISA, IHC, and/or RTQuIC technologies and results secured with MN-QuIC ${ }^{\mathrm{TM}}$ were $100 \%$ consistent with these tests. We conclude that hybrid AuNP and QuIC assays, such as MN-QuIC TM, have great potential for sensitive, field-deployable diagnostics of a variety of protein misfolding diseases.
\end{abstract}

\title{
Statement of Significance
}

Portable diagnostic tools for the detection of prion and protein-misfolding diseases are limited. Using chronic wasting disease (CWD) as a model, we demonstrate the functionality of a gold nanoparticle (AuNP)-based assay for the field-based detection of prion diseases. CWD is spreading through cervid 
populations across North America and the disease poses significant threats to a number of economic sectors, with global implications. Our newly developed diagnostic assay leverages the visible color change of AuNPs to detect the presence of CWD-causing prions. The assay can be performed using lowcost equipment and reagents, thus having great potential to expand prion disease surveillance efforts and prevent infectious prions from entering the human food chain.

Keywords: colorimetric assay, Chronic Wasting Disease, CWD, gold nanoparticle, MN-QuIC, neurodegenerative disease diagnostics, proteopathy, surveillance, transmissible spongiform encephalopathy

\section{Introduction}

A common feature of many neurodegenerative diseases is the presence of misfolded proteins that accumulate primarily within the central nervous system, ultimately contributing to advanced neurodegeneration and death. Misfolded protein diseases (proteopathies) impact a wide variety of mammals, including bovine spongiform encephalopathy (BSE) in cattle, scrapie in sheep, pituitary pars intermedia dysfunction in horses, chronic wasting disease (CWD) in cervids, Creutzfeldt-Jakob disease (CJD), Alzheimer's disease, and Parkinson's disease in humans (1-5). Given well-documented diagnostic limitations surrounding proteopathies in both animals and humans, (i.e., poor sensitivity, limited antibodies for immunobased assays, etc.), it is imperative that novel or next-generation diagnostic assays be developed in order to improve detection $(2,6-11)$. With respect to prion diseases (a proteopathy subclass caused by infectious proteins), tests that could be deployed in a variety of settings (e.g., hospitals, veterinary clinics, field stations, etc.) would greatly aid the detection of infectious prions thus limiting their spread. It is within this framework that we approach the development of diagnostic tools for CWD of cervids, a model neurodegenerative disorder with immediate needs for advanced, portable, diagnostic assays for effective surveillance. 
Like BSE in cattle or CJD in humans, CWD is a prion disease or Transmissible Spongiform Encephalopathy (TSE) (1, 12). CWD impacts cervids across North America, Scandinavia, and South Korea (e.g., elk, moose, mule deer, white-tailed deer, reindeer). The disease continues to spread to new populations, and there are increasing health concerns for both humans and animals exposed to CWD prion strains (13-15). All mammals have normal functioning cellular prion protein $\left(\operatorname{PrP}^{\mathrm{C}}\right)$ distributed throughout various tissues and playing essential roles in a variety of physiological functions, especially those of the central nervous system (16). Like other misfolding neurodegenerative disorders, $\operatorname{PrP}^{\mathrm{C}}$ in cervids adopts pathogenic conformations of misfolded prions $\left(\mathrm{PrP}^{\mathrm{CWD}}\right)$. $\mathrm{PrP}^{\mathrm{CWD}}$ propagates throughout an infected animal, forming fibrils that accumulate in lymph and nervous tissues, leading to death years after exposure. CWD poses obvious risks to the health of impacted cervid populations globally and the disease is an immediate threat to not only cervid health, but also all cervid-related economies. Indeed, cervid hunting and related activities generate tens of billions of USD annually in the United States (17). Across the world, cervids provide venison and a wide array of economically important products that are routinely consumed and/or used by humans (i.e., antler velvet, antlers, hides, etc.).

Current diagnostic methods for the detection of protein-misfolding diseases, including CWD and other TSEs, are limited (2, 6-11). Commonly utilized TSE diagnostic assays rely heavily on antibodybased enzyme-linked immunosorbent assay (ELISA) and immunohistochemistry (IHC) technologies that are expensive, time consuming, and require substantial training and expertise to operate (6). A feature of ELISA and IHC assays is that the antibodies routinely used are unable to differentiate between normal PrP and misfolded TSE-associated prion proteins $\left(\operatorname{PrP}^{\mathrm{TSE}}\right)$ thus requiring enzymatic digestion to enrich for $\operatorname{PrP}^{\mathrm{TSE}}$, a methodology that may impact diagnostic sensitivity through the destruction of particular TSE affiliated PrP strains $(18,19)$. Collectively, these antibody-based assays are limited in the identification of early stage TSE infections and they are primarily used on tissues collected post-mortem. The detection of prion seeding-activity was recently enhanced by various assays involving the amplification of protein misfolding in vitro, including protein misfolding cyclic amplification (PMCA) $(6,20)$, end-point quaking- 
induced conversion (EP-QuIC) $(21,22)$ and real-time quaking-induced conversion (RT-QuIC) $(6,23-26)$. Of these prion amplification methods both EP-QuIC and RT-QuIC utilize a recombinant mammalian PrP substrate $(\mathrm{rPrP})$ that is incubated and shaken with the diagnostic samples. When $\mathrm{PrP}^{\mathrm{TSE}}$ is present within a given QuIC reaction it induces a conformational change of the rPrP, forming a beta-sheet enriched mixture that is quantified with fluorescent Thioflavin T (ThT) measurements. Despite the advantages of EP-QuIC and RT-QuIC, there still remain major limitations; including the need for expensive and large laboratory equipment and complex strategies for visualizing and analyzing results. In short, more effective TSE diagnostic methods that leverage small and portable equipment with straightforward results, are needed to rapidly detect various TSEs and prevent additional spread and introduction of TSE prions into human and animal food chains. This is especially true for CWD, as the disease continues to expand across both farmed and wild cervid populations.

In parallel to diagnostic advancements based on prion and protein amplification methods, gold nanoparticles (AuNPs) have been increasingly used for diagnostic applications including drug delivery monitoring (27), disease detection (28), drinking water safety (29), and food safety (30). Due to localized surface plasmon resonances (density fluctuation of conduction electrons), AuNPs have optical absorption peaks that are extremely sensitive to the environment at the AuNP's surface (31-33). These unique optical properties make nanoparticles useful in color-based detection assays $(34,35)$. Previous studies have indicated affinities of prion proteins with a variety of bare and functionalized metals, including gold (35-38). In light of these observations we sought to investigate the diagnostic utility of gold nanoparticles for detecting $\operatorname{PrP}^{\mathrm{CWD}}$ within QuIC amplified products of CWD positive and negative white-tailed deer (WTD) tissues. By combining the unique color properties of AuNPs and the methods of quaking-based prion protein fibril amplification, we have produced a nanoparticle-based assay (herein named MinnesotaQuIC; MN-QuIC) that can detect the presence or absence of $\operatorname{PrP}^{\mathrm{CWD}}$ using both visual and spectroscopic methods (Fig. 1). This method uses only basic lab equipment that allows it to be deployed outside the laboratory. In March of 2019, we deployed the MN-QuIC assay to a field-station in rural southeastern 
bioRxiv preprint doi: https://doi.org/10.1101/2021.11.22.469560; this version posted November 22, 2021. The copyright holder for this preprint (which was not certified by peer review) is the author/funder, who has granted bioRxiv a license to display the preprint in perpetuity. It is made available under aCC-BY-NC-ND 4.0 International license.

Minnesota where the Minnesota Department of Natural Resources (DNR) was performing their annual CWD surveillance and culling efforts. We demonstrated proof of concept experiments for MN-QuIC's utility of a portable prion assay by successfully detecting CWD infected WTD tissues at the DNR fieldstation. 


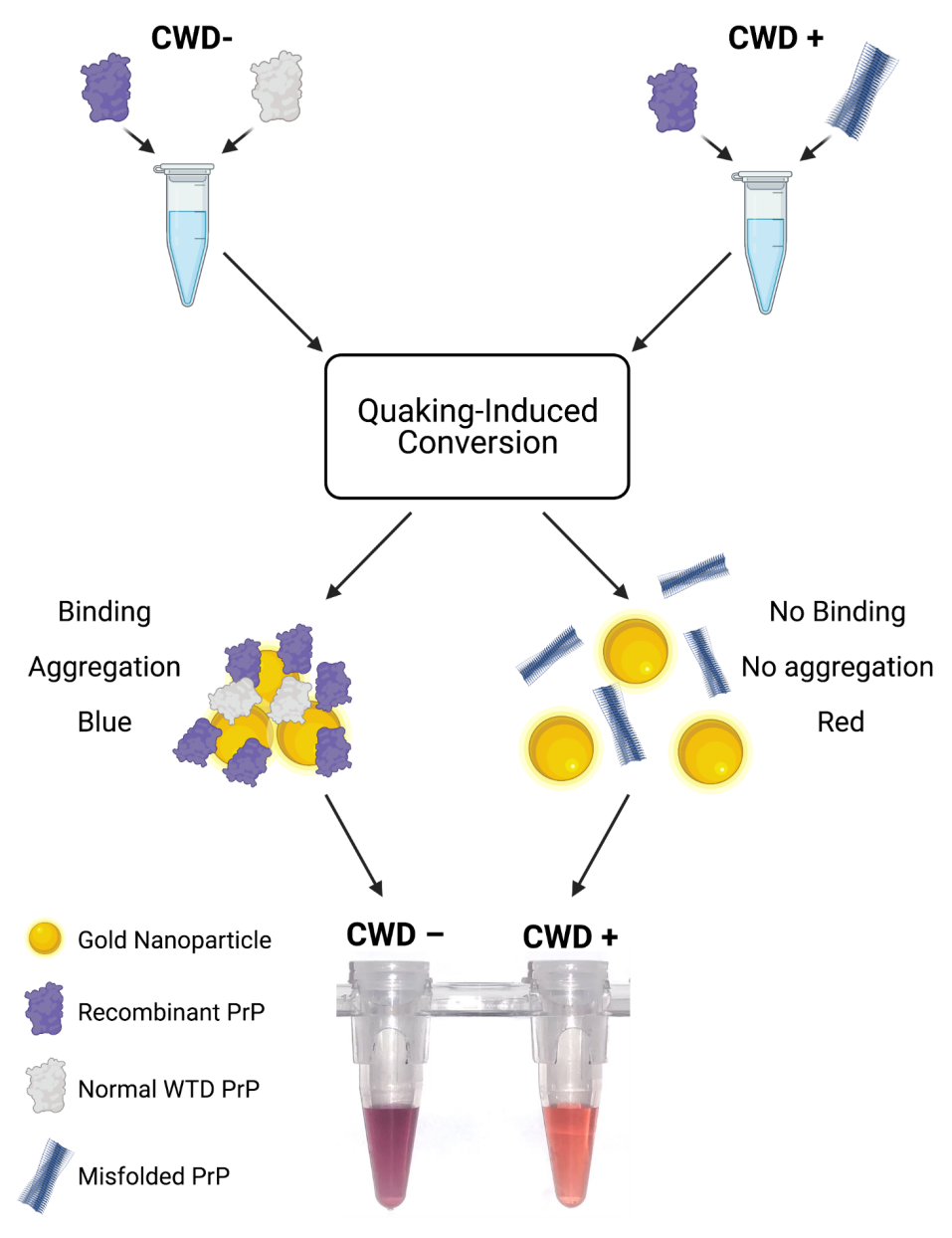

Figure 1. Overview of the MN-QuIC assay. Misfolded chronic wasting disease (CWD) prion seeds originating from biological samples of cervids are added to rPrP solutions. These solutions are then shaken and incubated for approximately 24 hours. If present, $\mathrm{PrP}^{\mathrm{CWD}}$ induces conformational changes of the $\mathrm{rPrP}$. Resulting products are diluted and added to an AuNP solution. CWD positive samples result in a red solution (peak absorbance wavelength $\sim 516 \mathrm{~nm}$ ) while CWD negative solutions are purple (peak absorbance wavelength $\sim 560 \mathrm{~nm})$. 


\section{Results}

\section{Comparison of gold nanoparticle interaction with recombinant cellular prions versus}

\section{amplified misfolded fibrils}

To investigate whether AuNPs can differentiate between misfolded PrP fibrils and normal PrP originating from recombinant hamster prion protein $(\mathrm{rHaPrP})$, two sets of reaction mixtures seeded with and without spontaneously misfolded rHaPrP prion fibrils were processed following modified RT-QuIC protocols without $\operatorname{ThT}(39,40)$. The presence of fibril formation was examined in all reaction mixtures by adding and quantifying ThT post hoc (Fig. 2a). ThT fluorescence was significantly different between misfolded $\mathrm{rHaPrP}$ (seeded) and native non-misfolded rHaPrP (no seed) ( $\mathrm{p}=0.05$; Fig. 2a). We hypothesized that misfolded prion fibrils would interact differently with citrate capped AuNPs, as compared with native $\mathrm{rHaPrP}$, and that the interaction would influence AuNP aggregation as measured by dynamic light scattering (DLS). Following ThT quantification, misfolded rHaPrP samples and native rHaPrP samples were spiked into separate AuNP solutions. After a 30-min incubation period at ambient temperature, there was a visible color difference between the AuNP solutions spiked with misfolded and native rHaPrP similar to the change in Fig. 1. This was also reflected in the visible spectrum of the absorbance peak (Fig. 2b). Color changes due to aggregation have been reported in literature for a variety of nanoparticle/protein combinations including prions and AuNPs $(34,35)$. To quantify this, DLS experiments were performed on three AuNP solutions (seeded, non-seeded, and blank; see Methods) and average effective particle size was determined for each sample (Fig. 2c). We observed a significant difference of AuNP effective particle sizes between AuNP solutions seeded with misfolded rHaPrP versus native non-seeded $\mathrm{rHaPrP}(\mathrm{p}=0.05)$ (Fig. 2c). The AuNPs spiked with no protein (blank) and misfolded rHaPrP exhibited similar particle size distributions (Fig. 2d,e), indicating that the misfolded rHaPrP solutions did not induce aggregation. On the contrary, the addition of diluted native rHaPrP resulted in larger particle sizes for AuNPs (Fig. 2f) than AuNPs with no protein added (Fig. 2d), indicating that the 
addition of diluted native rHaPrP caused AuNPs to aggregate. These results indicate differential AuNP binding capacity between native $\mathrm{rHaPrP}$ and misfolded $\mathrm{rHaPrP}$ fibrils.

a

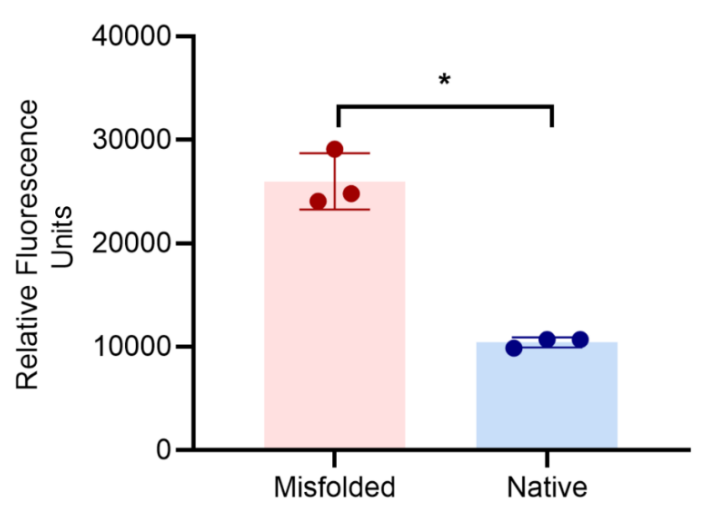

b

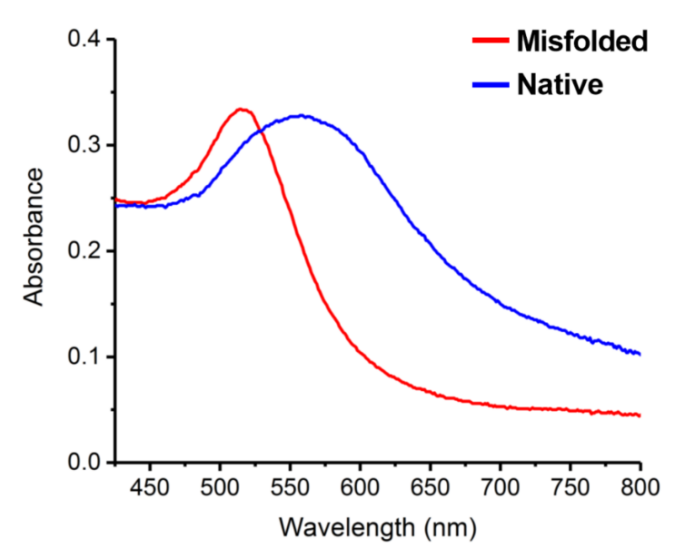

C

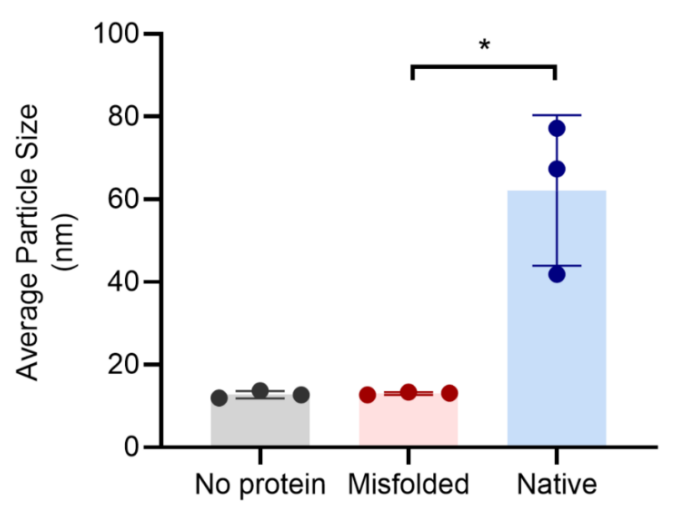

d

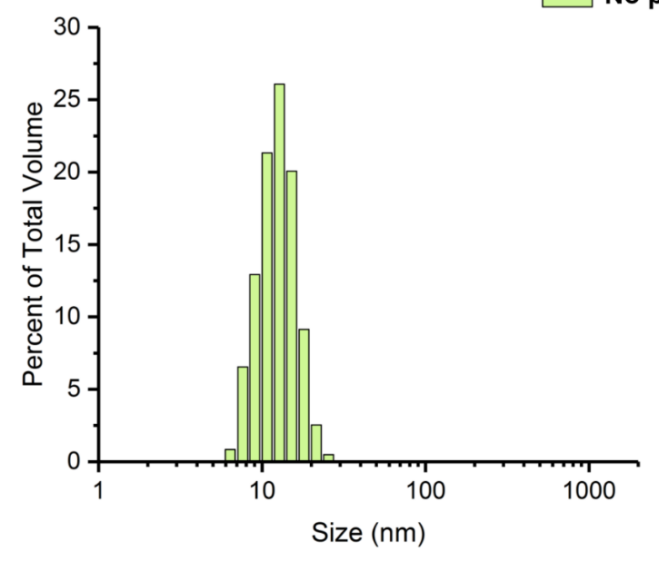

e

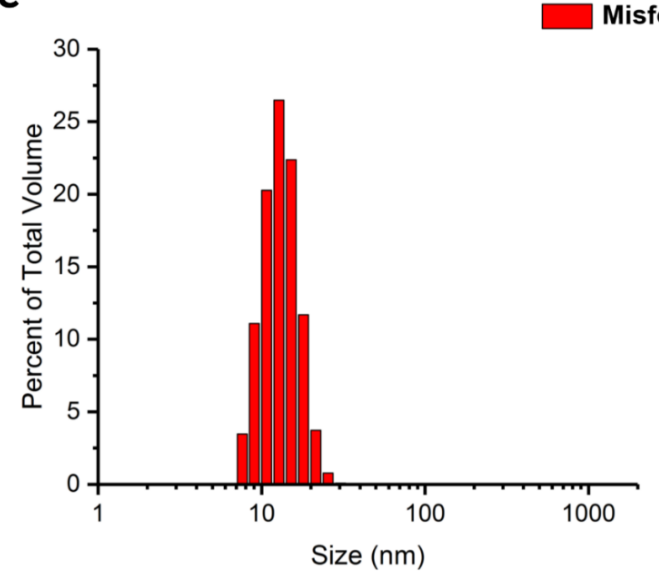

f

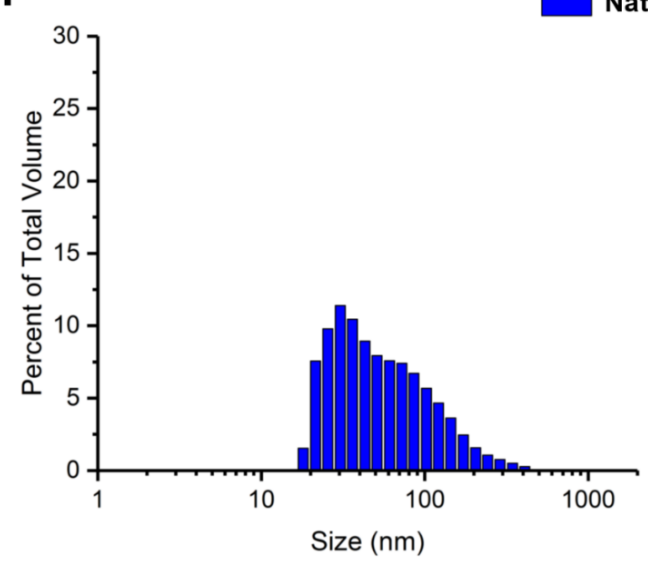




\section{Figure 2. ThT fluorescence, light absorbance, and average particle size measurements for native} and misfolded recombinant hamster prion protein. a. The relative fluorescence units (ThT fluorescence) of post QuIC solutions containing misfolded protein seeds and solutions without misfolded protein seeds. b. The absorbance spectrum of AuNP solutions spiked with misfolded rHaPrP from seeded QuIC reactions (red line) and non-misfolded/native rHaPrP from QuIC reactions without seed (blue line). c. Average effective particle sizes in AuNP solutions containing no protein, misfolded $\mathrm{rHaPrP}$, and native rHaPrP observed by dynamic light scattering reading (DLS). d. Distribution of DLS readings of AuNP solution with no protein added. Reported as percent of total volume of AuNPs present. e. Distribution of DLS readings of AuNP solution with misfolded rHaPrP. f. Distribution of DLS readings of AuNP solution with native rHaPrP. *, p-value $<0.05$, error bars show standard deviation.

\section{CWD positive and negative samples produce unique AuNP optical signatures}

Understanding that pathogenic prions (e.g., $\mathrm{PrP}^{\mathrm{Sc}}$ ) can induce $\mathrm{rHaPrP}$ misfolding and amplification (41) and thus influence AuNP aggregation (see above), we then investigated the potential of MN-QuIC for CWD diagnostics using $\operatorname{PrP}^{\mathrm{CWD}}$ positive and negative WTD lymphoid tissues. We used homogenates of independently confirmed CWD positive and negative WTD medial retropharyngeal lymph nodes (RPLN) (Table S1). Independent RT-QuIC analyses were performed on all tissues used for AuNP analyses (Fig. 3a) (26). Thermomixers have been used previously in conjunction with end-point ThT readings (i.e., EP-QuIC) to determine the presence of CJD prion seeding activity (22). In light of our previous results, we anticipated that AuNPs could be utilized to facilitate direct visualization of QuICamplified misfolded rHaPrP solutions that were seeded with CWD positive tissue using a standard benchtop thermomixer. To test this hypothesis, the same set of samples were added to RT-QuIC mastermix without ThT on 96-well plates, which were then subjected to shaking/incubation cycles on a thermomixer for $24 \mathrm{hrs}$. The post-amplification solutions were then diluted to $50 \%$ and added to AuNP solutions. We found that we were able to clearly distinguish CWD positive and negative samples simply through color difference appreciable by naked eye; the QuIC-amplified CWD positive and negative samples were red 
and purple, respectively (Fig. 3b). In order to quantify our observations and measure statistical differences, the absorbance spectrum of the AuNPs was measured from 400-800 nm using a 96-well plate reader. In the resulting absorbance spectrum, AuNP solutions combined with QuIC-amplified CWD positive samples had absorbance peaks near $516 \mathrm{~nm}$ (Fig. 3c), similar to the $515 \mathrm{~nm}$ absorbance peak of the AuNPs prior to the addition of protein solutions. However, the negative sample absorbance peaks were shifted to longer wavelengths of approximately $560 \mathrm{~nm}$ (Fig. 3c), confirming that the purple color of AuNP solutions from QuIC products originating from CWD negative tissue samples was consistent with the observed purple color of AuNP aggregates associated with native rHaPrp (Fig 2c,f). Accordingly, the peak AuNP absorbance wavelength of CWD negative samples are significantly larger $(\mathrm{p}<0.05)$ than CWD-positive samples (Fig. 3c). 


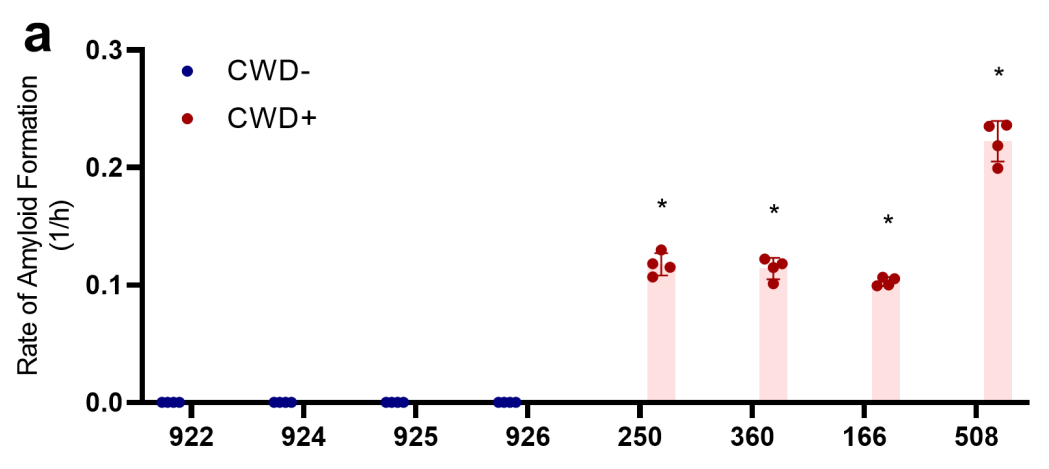

b
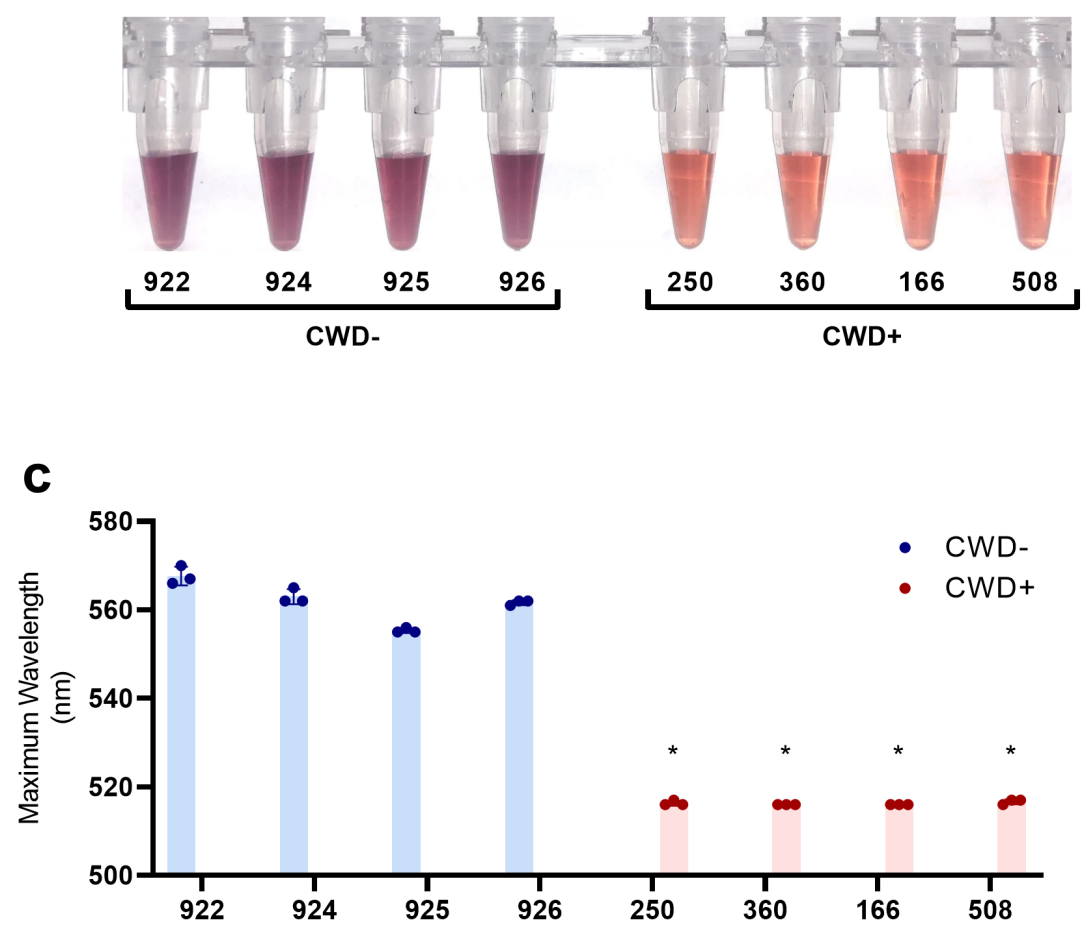

Figure 3. Comparison of RT-QuIC and MN-QuIC results for CWD positive and negative tissues. a.

RT-QuIC data for the rate of amyloid formation for negative and positive medial retropharyngeal lymph node tissue samples from wild white-tailed deer. Sample identification number on horizontal axis. b. Photo of MN-QuIC tubes showing the color difference for the same set of tissue samples used in panel a. c. MN-QuIC peak absorbance wavelength of the same set of solutions used in panel b. ${ }^{*}$, p-value $<0.05$, error bars show standard deviation. 


\section{Electrostatic forces play a role in AuNP CWD detection}

Considering the results described above, we aimed to determine the potential mechanism underlying AuNP aggregation caused by rHaPrP solutions. Studies with prions and other proteins have shown that electrostatic forces help govern the interactions between nanoparticles and proteins $(35,45$, 46). Because the theoretical isoelectric point (IP) of our $\mathrm{rHaPrP}$ is around $\mathrm{pH} 8.9$ (47), $\mathrm{rHaPrP}$ is positively charged in the pH7.4 AuNP buffer whereas citrate capped AuNPs are negatively charged even at pHs well below our buffer (48). Thus at $\mathrm{pH} 7.4$, there exists an electrostatic attractive force between AuNPs and native rHaPrP that contributes to their interactions (aggregation and the color change). We predicted that the charge on the protein would change when the $\mathrm{pH}$ of the environment is altered and that the interaction between AuNP and $\mathrm{rHaPrP}$ would be disrupted. This prediction was based on the rationale that when the $\mathrm{pH}$ of a solution is raised closer to the IP of the rHaPrP, the charge of the protein will become closer to neutral, decreasing the force of attraction between AuNP and rHaPrP. We showed that as the $\mathrm{pH}$ of the AuNP solution was raised closer to the IP of rHaPrP, the absorbance peak shift from 530 nm of the AuNP-rHaPrP solution decreased (Fig. 4) while the control AuNP solution with no protein had very little peak deviation from $515 \mathrm{~nm}$. This suggests that electrostatic interactions were at least partially responsible for facilitating the $\mathrm{rHaPrP}$ interactions with AuNPs. QuIC-amplified $\mathrm{PrP}^{\mathrm{CWD}}$ products, on the other hand, have experienced major conformational changes from its native form (as confirmed by ThT beta-sheet binding; Fig. 3) and have formed aggregates. We hypothesized that this conformational change/aggregation reduced electrostatic attraction thus AuNP solutions with $\operatorname{PrP}^{\mathrm{CWD}}$ would remain around $515 \mathrm{~nm}$ (red) for all tested solutions (Fig. 4). 


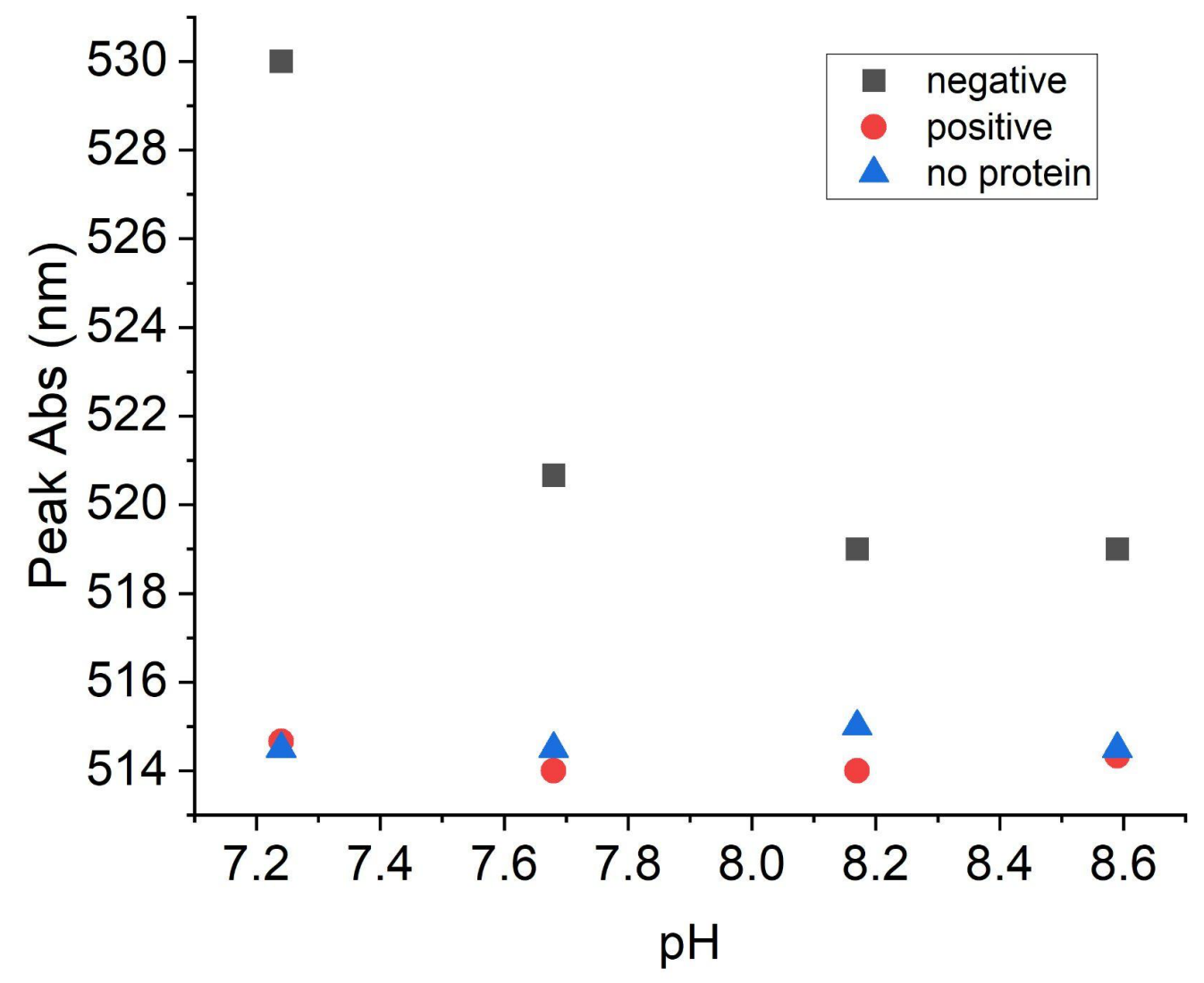

Figure 4. Wavelength of peak absorbance of AuNPs in different $\mathrm{pH}$ buffers containing native CWD negative $\mathrm{rHaPrP}$ (square), $\mathrm{PrP}^{\mathrm{CWD}}$ positive (circle), and blank/no protein (triangle) solutions. 


\section{Field deployment and higher throughput protocols}

To show the potential for a portable, field-deployable diagnostic we performed proof of concept experiments at a rural Minnesota DNR field-station. We tested both pooled and individual tissues consisting of retropharyngeal lymph nodes, parotid lymph nodes and palatine tonsils tissues from 13 WTD that the DNR had recently collected from the surrounding wild deer population. Three of these animals (blinded to the field team) were CWD positive as determined by regulatory ELISA and IHC testing of medial retropharyngeal lymph nodes. Using a blinded testing approach, MN-QuIC successfully detected, via red AuNP solutions, all three CWD positive animals. We also secured CWD not detected or negative results (purple AuNP solutions) for the 10 animals that were independently identified by ELISA as CWD not detected (as determined by regulatory ELISA testing, Table S2). These proof-of-concept experiments demonstrate the potential utility of MN-QuIC as a portable, field-deployable diagnostic tool for researchers and agencies.

In order to demonstrate higher throughput protocols, palatine tonsil samples from a set of ten CWD negative and ten CWD positive white-tailed deer (Table S3) were tested in a 96-well format. The status of these tissues was independently confirmed with RT-QuIC (Fig S1a). For MN-QuIC, each sample had eight replicates and was prepared and subjected to the QuIC protocol using a 96-well plate on a thermomixer for 24hrs. A multichannel pipette was then used to add the QuIC amplified protein to a separate 96-well plate filled with AuNP solution. Color changes were observed within the first minute. For RT-QuIC analyses, it is common practice to consider a particular sample positive if $50 \%$ or more of its wells are positive $(23,42-44)$. Using this approach, we successfully identified all 10 CWD positive animals using MN-QuIC (Fig. S1b). CWD negative samples were identified using a threshold of $<50 \%$ of wells being red (i.e., majority purple in color) and we correctly identified $100 \%$ of CWD negative samples with the MN-QuIC assay (Fig. S1b). In addition to visual color, these results were assessed by investigating the peak shift from the expected $517 \mathrm{~nm}$ (red) for positive samples and all red wells had absorbance peaks within $4 \mathrm{~nm}$ of $517 \mathrm{~nm}$. 


\section{Discussion}

Given the continued spread of CWD among cervid populations throughout North America, Northern Europe, and South Korea $(13,14)$ there is an urgent need to develop novel diagnostic tools for CWD. Here, we combined QuIC amplification of CWD prions with the simplicity of citrate-capped gold nanoparticles for the visualization of positive vs. negative CWD lymph node and palatine tonsil WTD samples. We hypothesized that the large conformational differences between the rHaPrP substrate and misfolded prion fibrils would influence the aggregation of AuNPs in solution. By injecting post QuICamplified protein solutions into AuNPs, we were able to clearly distinguish via visible color change between CWD positive and CWD negative medial retropharyngeal lymph node and palatine tonsil tissues. AuNP solutions for these samples appeared red and purple, respectively. We further confirmed that the color change was a result of the aggregation of gold nanoparticles by conducting DLS that compared the effective particle sizes in the presence of native and misfolded $\mathrm{rHaPrP}$. We hypothesized that the observed AuNP aggregation was governed by electrostatic interactions, and thus we altered the $\mathrm{pH}$ of the AuNP solution and observed that the aggregation states of CWD-negative samples are altered with increasing $\mathrm{pH}$. Finally, we demonstrated proof of concept experiments for the real-world utility of MNQuIC by successfully identifying CWD infected WTD tissues at a rural DNR field station in southeastern MN (Rushford, MN). In total, we examined 13 CWD positive and 24 CWD not-detected WTD that were independently tested using ELISA, IHC, and RT-QuIC technologies and results secured with MNQuIC TM $^{\text {Tere }} 100 \%$ consistent with these tests.

The primary laboratory equipment for MN-QuIC consists of a tissue homogenizer, temperaturecontrolled shaker, and, if desired (but not necessary), a spectrometer for light absorbance readings in addition to visual observations. Compared to RT-QuIC and PMCA, which leverage ThT fluorescence and antibody-based Western blotting, respectively (6), results from MN-QuIC can be visualized with the naked eye and quantified using straightforward light-absorbance readings. Because MN-QuIC is a protein amplification method, it can be adapted to use any tissue or biological sample that existing and future RT- 
QuIC protocols use, thus giving MN-QuIC wide versatility. Recent publications have used specially functionalized AuNPs to detect a variety of protein targets $(35,49-51)$. However, the AuNPs used here are capped with citrate, which is one of the most common methods for stabilization making it simple and widely commercially available.

AuNPs have been used in a variety of advanced sensing applications $(27-30,52,53)$. Our work demonstrates that AuNPs can open new and promising avenues for the straightforward identification of misfolded prions. Because prion proteins have strong interactions with simply functionalized metallic surfaces, besides AuNPs, we envision a broad range of metallic nanoparticles with various materials and shapes to be useful in detection. Additionally, substrate-based nanostructures exhibiting optical resonances could be useful in detecting conformational changes via other sensing modalities such as surface-enhanced infrared absorption spectroscopy (54) to further improve the speed and accuracy of prion detection.

To demonstrate field deployment capabilities of the MN-QuIC assay, we collected medial retropharyngeal lymph nodes, parotid lymph nodes and palatine tonsil samples from 13 WTD at a rural DNR field station. Using both pooled and individual tissues of these 13 individuals, MN-QuIC was 100\% successful in identifying three CWD positive and 10 CWD not-detected animals. The successful fieldbased classification of these animals provides clear proof of concept demonstration of MN-QuICs utility as a portable, sensitive field test. We note that our MN-QuIC analyses have yet to produce statistically significant false-negative results. This observation is critically important when considering MN-QuIC as a field-based diagnostic tool for CWD. Moreover, any positive result can be independently validated using downstream RT-QuIC, ELISA, and/or IHC testing. Given growing concerns of CWD prion strain variation and risks to human and animal health (15), any field-based diagnostic assay that avoids producing false-negative is preferred.

RPLN and palatine tonsils collected from WTD were the basis for the laboratory based analyses conducted herein because these tissues are ideal for early and accurate identification of CWD infection, with tonsils additionally having ante-mortem applications $(26,55)$. Future analyses will focus on 
leveraging MN-QuIC for CWD diagnostics using a variety of antem-mortem biological samples (e.g., blood, saliva, and feces). RT-QuIC amplification assays using samples acquired from living deer have recently been reported $(40,56)$ and these assays could readily be combined with MN-QuIC to provide field-deployable ante-mortem tests of both wild and farmed cervids. Moreover, it is possible that MNQuIC has potential food-safety test applications given the recent documentation of RT-QuIC-based detection of CWD prions in WTD muscles used for human and animal consumption (25).

Additional research is needed to clearly identify the mechanisms underlying the AuNP and prion interaction reported herein. In this study, we hypothesized that as the $\mathrm{pH}$ approaches the prion's isoelectric point, the electrostatic force of attraction between the negatively charged citrate capped AuNP and the protein would decrease. Our analyses revealed that as the $\mathrm{pH}$ neared the theoretical isoelectric point of rHaPrP, the wavelength of the peak absorbance of the AuNPs spiked with native (CWD negative) protein decreased. We also found that $\mathrm{pH}$ alterations had little effect on AuNP solutions with QuICamplified CWD positive seeds and solutions without protein, indicating that the effect of $\mathrm{pH}$ on native rHaPrP-spiked AuNP solutions was not caused by intrinsic AuNP aggregation in response to the changing pH. Therefore, we concluded that the interactions were at least partially governed by electrostatic forces. However, other factors such as hydrophobic interactions $(57,58)$ or the structural impact of fibril formation on AuNP binding could also play a role. Various studies have shown that both native and misfolded PrP bind various metal ions and bulk metals including gold (16, 36-38). However, our research reveals AuNPs stabilized with a simple citrate capping readily interact with the truncated $\mathrm{rHaPrP}$ substrate that is used as the primary substrate for a growing variety of QuIC assays, but not the misfolded, beta-sheet enriched form of PrP. Future structural analyses focused on native rHaPrP and prion fibrils (59) could provide further insight into how native rHaPrP, but not misfolded PrP fibrils, influence citratecapped AuNP aggregation.

We postulate that the MN-QuIC assay holds great promise not only for the visual detection of CWD-positive samples but also the detection of other protein misfolding diseases. The need for inexpensive, sensitive, widely deployable diagnostics for neurodegenerative diseases is only growing as 
neurodegenerative diseases are predicted to greatly increase in the next decades $(2,60)$. Future research should focus on developing AuNP-based assays for other neurodegenerative diseases. It is likely that advances in CWD diagnostics will yield technologies that are useful for a broad range of neurodegenerative diseases (6). A critical observation is that QuIC protocols have been developed for a variety of protein misfolding diseases including scrapie in sheep, BSE in cattle, and Alzheimer's, Parkinson's, and CJD in humans $(21,43,61-63)$. Thus, we posit that the combination of AuNP technology with QuIC-based assays has great potential for the development of versatile neurodegenerative disease diagnostic platforms.

\section{Methods}

\section{Tissue preparation}

Twenty-eight WTD tissues (14 CWD-negative and 14 CWD-positive) were selected for laboratory RTQuIC and MN-QuIC analyses. These samples were collected from WTD through collaboration with the Minnesota Department of Natural Resources (Schwabenlander et al. 2021; Tables S1 and S3) and their CWD status was independently identified utilizing the Bio-Rad TeSeE Short Assay Protocol (SAP) Combo Kit (BioRad Laboratories Inc., Hercules, CA, USA). Positive RPLNs were confirmed by IHC at the Colorado State University Veterinary Diagnostic Laboratory (CSU VDL). Metadata containing information of all specimens examined in the lab, including tissue type, is provided in supplementary materials (Tables S1 and S3). WTD RPLNs and palatine tonsils were homogenized in PBS (10\% w:v) in $2 \mathrm{~mL}$ tubes containing $1.5 \mathrm{~mm}$ zirconium beads with a BeadBug Homogenizer (Benchmark Scientific, Sayreville New Jersey, USA) on max speed for $90 \mathrm{sec}$. All CWD positive and negative samples were selected based on independant ELISA, IHC, and/or RT-QuIC results and were subsampled using methods as reported in Schwabenlander et al. (26) 


\section{Preparation of Recombinant Substrate}

Recombinant hamster PrP (HaPrP90-231) production and purification followed the methods in Schwabenlander et al. (26). The substrate is derived from a truncated form (amino acids 90-231) of the Syrian hamster PRNP gene cloned into the pET41-a(+) expression vector and was expressed in Rosetta (DE3) E. coli. The original clone was provided by the National Institutes of Health Rocky Mountain Laboratory.

\section{RT-QuIC for cervid lymph tissues and spontaneous misfolding of recombinant prion}

\section{protein}

For QuIC analysis, a master mix was made to the following specifications: $1 \mathrm{X}$ PBS, $1 \mathrm{mM}$ Ethylenediaminetetraacetic acid (EDTA), $170 \mathrm{mM} \mathrm{NaCl}, 10 \mu \mathrm{M}$ thioflavin $\mathrm{T}(\mathrm{ThT})$, and $0.1 \mathrm{mg} / \mathrm{mL}$ rHaPrP. In instances where the end reaction would be analyzed using AuNPs, ThT was excluded. The $10 \%$ tissue homogenates were further diluted 100-fold in $0.1 \%$ Sodium Dodecyl Sulfate (SDS) using methods from Schwabenlander et al. (26) (final tissue dilution: $0.1 \%$ ), and $2 \mu \mathrm{L}$ of the diluent were added to each well containing $98 \mathrm{uL}$ of RT-QuIC master mix. Spontaneous misfolding of recombinant prion protein was generated similarly but with unfiltered recombinant proteins and reagents. For these reactions, no infectious seed was necessary. The spontaneously misfolded material was used to seed reactions for the dynamic light scattering experiment, described below. Plates were amplified on a FLUOstar ${ }^{\circledR}$ Omega plate reader (BMG Labtech, Cary, North Carolina, USA; $42^{\circ} \mathrm{C}, 700 \mathrm{rpm}$, double orbital, shake for $57 \mathrm{~s}$, rest for $83 \mathrm{~s}$ ). Fluorescent readings were taken at $\sim 45 \mathrm{~min}$ increments.

\section{Thermomixer-based Amplification}

We leveraged a standard benchtop shaking incubator (thermomixer) to produce QuIC-based prion amplifications as previously reported by Cheng et al. (21) and Vendramelli et al (22), although with slight modifications. Plates which were made for amplification on the thermomixer were prepared identical to those amplified on the plate reader. Reactions were performed on a ThermoMixer ${ }^{\circledR}$ C equipped with 
SmartBlock plate and Thermotop (Eppendorf, Enfield, Connecticut, USA) at $48^{\circ} \mathrm{C}$ for $24 \mathrm{hrs}$ at $600 \mathrm{RPM}$ (60s shake and 60s rest). We selected a 24 hour run time based on independent RT-QuIC results for medial retropharyngeal lymph nodes and palatine tonsils from CWD positive WTD reported in Schwabenlander et al. (26), including those examined herein, showing significant seeding activity within 9 to 24 hours (Fig. S2). The resultant products were visualized with the addition of gold nanoparticles (as described below).

\section{Preparation of gold nanoparticles (AuNP)}

Post amplified material was visualized with $15 \mathrm{~nm}$ citrate capped gold nanoparticles purchased from Nanopartz (Loveland, Colorado, USA) with stock concentrations ranging from 2.45nM to $2.7 \mathrm{nM}$. AuNP protocols were modified from Špringer et al. (64) and Zhang et al. (35). AuNPs were buffer exchanged using 530ul of stock solution that was centrifuged in $1.6 \mathrm{~mL}$ tubes at $13,800 \mathrm{~g}$ for $10 \mathrm{~min} .490 \mathrm{ul}$ of supernatant was removed and the undisturbed pellet was resuspended with $320 \mathrm{ul}$ of a low concentration phosphate buffer $\left(\mathrm{PBS}_{\text {low }}\right.$; $\mathrm{pH} 7.4$ via addition of $\mathrm{HCl}$ ) made of $10 \mathrm{mM} \mathrm{Na}_{2} \mathrm{HPO}_{4}$ (Anhydrous), $2.7 \mathrm{mM}$ $\mathrm{KCl}, 1.8 \mathrm{mM} \mathrm{KH}_{2} \mathrm{PO}_{4}$ (Monobasic). After the quaking/incubation steps, protein solutions were diluted to $50 \%$ in MN-QuIC buffer, consisting of $1 \mathrm{X}$ PBS with the addition of final concentrations of $1 \mathrm{mM}$ EDTA, $170 \mathrm{mM} \mathrm{NaCl}, 1.266 \mathrm{mM}$ Sodium Phosphate. Forty microliters of the protein diluted $50 \%$ in MN-QuIC buffer was then added to the 360ul AuNP solution with ample mixing (results shown in Fig 3b,c). This solution was left to react at room temperature (RT) for $30 \mathrm{~min}$ (although a visible color change is observable within $60 \mathrm{sec}$ ) before visual color was recorded (purple or red) and photographed. After images were taken, three replicates of 100ul were taken from the 400ul AuNP mixture and pipetted into three separate wells of a 96-well plate. The absorbance spectrum was then recorded for each well at wavelengths 400-800nm using the FLUOstar ${ }^{\circledR}$ Omega plate reader (BMG Labtech, Cary, North Carolina, USA). For AuNP visualization experiments performed to determine higher throughput capacity (Fig S1), proteins were prepared in the same way. After amplification on the thermomixer, proteins on a 96-well plate were diluted to $50 \%$ using MN-QuIC buffer and a multichannel pipette. Ninety microliters of 
AuNPs were then added to a separate non-binding 96-well plate. The AuNP wells were spiked with 10ul of the diluted protein from the thermomixer (post-amplification) using a multichannel pipette. After waiting 30min, the color changes were observed and the absorbance spectrum of the plate was taken.

\section{Dynamic light scattering}

Spontaneously misfolded $\mathrm{rHaPrP}$ samples (described above) were produced from solutions of $\mathrm{rHaPrP}$ with no seed added. In addition to these samples, a 96-well RT-QuIC reaction was performed with half the wells consisting of native rHaPrP seeded with spontaneously misfolded protein, and half consisting of native $\mathrm{rHaPrP}$ with no seed. The 96-well plate was then amplified using QuIC protocols described above. Post amplification, seeded samples were confirmed to have beta-sheet fibrillation while the non-seeded samples were confirmed to not have fibrillation based on ThT binding (described above). Seeded and non-seeded samples were diluted to 50\% in MN-QuIC buffer and 40ul of these solutions were added to 360ul of AuNPs in PBS low. Additionally, a blank with no protein was produced by adding 40ul of MNQuIC buffer to 360ul of AuNPs in $\mathrm{PBS}_{\text {low }}$. For native rHaPrP samples, color change could start to be observed within 1 min of protein addition. No color change was observed in spontaneously misfolded rHaPrP samples at any time length. Dynamic light scattering measurements of all samples were taken after 5 min of protein addition using a Microtract NanoFlex Dynamic Light Scattering Particle Analyzer (Verder Scientific, Montgomeryville, PA, USA) and measurement times were 60 seconds. Five measurements were taken for each sample and then averaged.

\section{Effects of pH on the AuNP-protein interaction}

In order to test the effects of $\mathrm{pH}$ on protein interacting with AuNP, four different 10mM tris-buffer solutions with pHs ranging from 7.2 to 8.6 were created. Tris was used to give buffering for the desired $\mathrm{pH}$ range. AuNPs were buffer exchanged as described above except tris-buffer was used instead of $\mathrm{PBS}_{\text {low. }}$ Protein solutions were added as previously described. 


\section{Additional statistical information}

GraphPad Prism version 9.0 for Windows (GraphPad Software, San Diego, California USA,

www.graphpad.com) was used for conducting statistical analysis. Three technical replicates were used to demonstrate the potential application of AuNP on spontaneously misfolded rHaPrP. For initial trials on RPLN tissues from eight (four positive and four negative) animals, four and three technical replicates used for RT-QuIC and AuNPs respectively. For plate based protocols, we tested palatine tonsils from ten positive and ten negative animals using four and eight replicates for RT-QuIC and AuNPs respectively. Unless specified in figures, rate of amyloid formation and maximum wavelength of samples were compared to negative controls on the respective plate. The one-tailed Mann-Whitney unpaired u-test $(\alpha=0.05)$ was used to test the average difference for all parameters of interests between samples.

\section{Field deployment}

In March of 2021, we collaborated with the Minnesota Department of Natural Resources (DNR) during annual CWD surveillance of the wild WTD population in Fillmore and Winona Counties, Minnesota. We assembled the necessary MN-QuIC equipment as described above on two portable tables within a DNR facility in Rushford, MN. Medial retropharyngeal lymph nodes, parotid lymph nodes and palatine tonsil were collected as described in Schwabenlander et al. (26), sampled and pooled together for each of the 13 animals tested. Tissues for suspected positive animals were tested individually (Table S2). All tissues were subject to $24 \mathrm{hr} \mathrm{MN}-\mathrm{QuIC}$ protocols as described above. Three replicates were performed for each of the 13 animals and, for field-based analyses, an animal was considered CWD positive if one or more replicates was red. An animal was considered CWD not-detected if all three replicates were blue or purple. 


\section{Acknowledgements}

Dr. Christopher Ertsgaard and Dong-Jun Lee provided discussions on experimental results and protocols. Portions of this work were conducted in the Minnesota Nano Center, which is supported by the National Science Foundation through the National Nano Coordinated Infrastructure Network (NNCI) under Award Number ECCS-2025124. Fred Schendel, Tom Douville, and staff of the University of Minnesota Biotechnology Resource Center provided critical support with respect to large-scale production of recombinant proteins. We thank the Minnesota Department of Natural Resources, especially Michelle Carstensen, Erik Hildebrand, Patrick Hagen and Kelsie LaSharr, for providing the white-tailed deer tissues used for our analyses and logistical assistance for MN-QuIC field-deployment. Kathi Wilson of the Colorado State University Veterinary Diagnostic Laboratory kindly provided assistance with ELISA and IHC testing of samples reported herein. Suzanne Stone provided valuable logistical assistance with our molecular work. We thank NIH Rocky Mountain Labs, especially Byron Caughey, Andrew Hughson, and Christina Orru for training and assistance with the implementation of RT-QuIC and for supplying the rHaPrP clone. Funding for research performed herein was provided by the Minnesota State Legislature through the Minnesota Legislative-Citizen Commission on Minnesota Resources (LCCMR), Minnesota Agricultural Experiment Station Rapid Agricultural Response Fund, and start-up funds awarded to PAL through the Minnesota Agricultural, Research, Education, Extension and Technology Transfer (AGREETT) program. Fig. 1 was created using BioRender (BioRender.com).

\section{Conflict of Interest}

The authors declare no conflicts of interest. 


\section{References}

1. S. B. Prusiner, Nobel Lecture: Prions. Proc. Natl. Acad. Sci. 95, 13363-13383 (1998).

2. G. C. Telling, Breakthroughs in antemortem diagnosis of neurodegenerative diseases. Proc. Natl. Acad. Sci. U. S. A. 116, 22894-22896 (2019).

3. J. Collinge, Prion diseases of humans and animals: their causes and molecular basis. Annu. Rev. Neurosci. 24, 519-550 (2001).

4. E. S. Williams, S. Young, Chronic wasting disease of captive mule deer: a spongiform encephalopathy. J. Wildl. Dis. 16, 89-98 (1980).

5. J. S. Fortin, et al., Equine pituitary pars intermedia dysfunction: a spontaneous model of synucleinopathy. Sci. Rep. 11, 16036 (2021).

6. N. J. Haley, J. A. Richt, Evolution of Diagnostic Tests for Chronic Wasting Disease, a Naturally Occurring Prion Disease of Cervids. Pathogens 6 (2017).

7. N. J. Haley, et al., Chronic wasting disease management in ranched elk using rectal biopsy testing. Prion 12, 93-108 (2018).

8. B. Martinez, P. V. Peplow, MicroRNAs in Parkinson's disease and emerging therapeutic targets. Neural Regeneration Res. 12, 1945-1959 (2017).

9. M. J. Hajipour, et al., Advances in Alzheimer's Diagnosis and Therapy: The Implications of Nanotechnology. Trends Biotechnol. 35, 937-953 (2017).

10. M. P. Figgie Jr, B. S. Appleby, Clinical Use of Improved Diagnostic Testing for Detection of Prion Disease. Viruses 13 (2021).

11. L. Parnetti, et al., CSF and blood biomarkers for Parkinson's disease. Lancet Neurol. 18, 573-586 (2019).

12. S. B. Prusiner, et al., Scrapie Prions Aggregate to Form Amyloid-Like Birefringent Rods. Cell 35, 349-358 (1983).

13. S. Hannaoui, H. M. Schatzl, S. Gilch, Chronic wasting disease: Emerging prions and their potential risk. PLoS Pathog. 13, e1006619 (2017).

14. D. O. Joly, et al., Chronic wasting disease in free-ranging Wisconsin White-tailed Deer. Emerg. Infect. Dis. 9, 599-601 (2003).

15. M. T. Osterholm, et al., Chronic Wasting Disease in Cervids: Implications for Prion Transmission to Humans and Other Animal Species. MBio 10 (2019).

16. L. Westergard, H. M. Christensen, D. A. Harris, The cellular prion protein (PrPC): Its physiological function and role in disease. Biochim Biophys Acta 1772, 629-644 (2007).

17. U.S. Fish and Wildlife Service \& U.S. Census Bureau, "2016 National Survey of Fishing, Hunting and Wildlife-Associated Recreation" (U.S. Fish and Wildlife Service, 2018). 
18. J. G. Safar, et al., Prion clearance in bigenic mice. J. Gen. Virol. 86, 2913-2923 (2005).

19. N. J. Haley, et al., Sensitivity of protein misfolding cyclic amplification versus immunohistochemistry in ante-mortem detection of chronic wasting disease. J. Gen. Virol. 93, 11411150 (2012).

20. G. P. Saborio, B. Permanne, C. Soto, Sensitive detection of pathological prion protein by cyclic amplification of protein misfolding. Nature 411, 810-813 (2001).

21. K. Cheng, et al., Endpoint Quaking-Induced Conversion: a Sensitive, Specific, and High-Throughput Method for Antemortem Diagnosis of Creutzfeldt-Jacob Disease. J. Clin. Microbiol. 54, 1751-1754 (2016).

22. R. Vendramelli, A. Sloan, S. L. R. Simon, D. Godal, K. Cheng, ThermoMixer-Aided Endpoint Quaking-Induced Conversion (EP-QuIC) Permits Faster Sporadic Creutzfeldt-Jakob Disease (sCJD) Identification than Real-Time Quaking-Induced Conversion (RT-QuIC). J. Clin. Microbiol. 56, e00423-18 (2018).

23. Y. C. Cheng, et al., Early and Non-Invasive Detection of Chronic Wasting Disease Prions in Elk Feces by Real-Time Quaking Induced Conversion. PLoS One 11, e0166187 (2016).

24. R. Atarashi, et al., Ultrasensitive human prion detection in cerebrospinal fluid by real-time quakinginduced conversion. Nat. Med. 17, 175-178 (2011).

25. M. Li, et al., RT-QuIC detection of CWD prion seeding activity in white-tailed deer muscle tissues. Sci. Rep. 11, 16759 (2021).

26. M. D. Schwabenlander, et al., Comparison of Chronic Wasting Disease Detection Methods and Procedures: Implications for Free-Ranging White-Tailed Deer (Odocoileus Virginianus) Surveillance and Management. J. Wildl. Dis. (2021) https:/doi.org/10.7589/JWD-D-21-00033.

27. C. Ruppert, N. Phogat, S. Laufer, M. Kohl, H.-P. Deigner, A smartphone readout system for gold nanoparticle-based lateral flow assays: application to monitoring of digoxigenin. Microchim. Acta 186, 119 (2019).

28. T.-T. Tsai, et al., Diagnosis of Tuberculosis Using Colorimetric Gold Nanoparticles on a PaperBased Analytical Device. ACS Sens 2, 1345-1354 (2017).

29. R. Thiramanas, R. Laocharoensuk, Competitive binding of polyethyleneimine-coated gold nanoparticles to enzymes and bacteria: a key mechanism for low-level colorimetric detection of gram-positive and gram-negative bacteria. Microchim. Acta 183, 389-396 (2016).

30. X.-J. Du, T.-J. Zhou, P. Li, S. Wang, A rapid Salmonella detection method involving thermophilic helicase-dependent amplification and a lateral flow assay. Mol. Cell. Probes 34, 37-44 (2017).

31. K. M. Mayer, J. H. Hafner, Localized surface plasmon resonance sensors. Chem. Rev. 111, 38283857 (2011).

32. V. Myroshnychenko, et al., Modelling the optical response of gold nanoparticles. Chem. Soc. Rev. 37, 1792-1805 (2008).

33. S. Lal, S. Link, N. J. Halas, Nano-optics from sensing to waveguiding. Nature Photonics 1, 641-648 (2007). 
34. W. Zhao, M. A. Brook, Y. Li, Design of gold nanoparticle-based colorimetric biosensing assays. Chembiochem 9, 2363-2371 (2008).

35. H.-J. Zhang, et al., Gold nanoparticles as a label-free probe for the detection of amyloidogenic protein. Talanta 89, 401-406 (2012).

36. E. Flechsig, et al., Transmission of scrapie by steel-surface-bound prions. Molecular Medicine 7, 679-684 (2001).

37. C. Weissmann, M. Enari, P. C. Klöhn, D. Rossi, E. Flechsig, Transmission of prions. J. Infec. Dis. 186, S157-S165 (2002).

38. K. M. Luhr, P. Löw, A. Taraboulos, T. Bergman, K. Kristensson, Prion adsorption to stainless steel is promoted by nickel and molybdenum. J. Gen. Virol. 90, 2821-2828 (2009).

39. D. M. Henderson, et al., Quantitative assessment of prion infectivity in tissues and body fluids by real-time quaking-induced conversion. J. Gen. Virol. 96, 210-219 (2015).

40. J. M. Tennant, et al., Shedding and stability of CWD prion seeding activity in cervid feces. PLoS One 15, e0227094 (2020).

41. D. M. Henderson, et al., Progression of chronic wasting disease in white-tailed deer analyzed by serial biopsy RT-QuIC and immunohistochemistry. PLoS One 15, e0228327 (2020).

42. N. J. Haley, D. M. Henderson, K. Senior, M. Miller, R. Donner, Evaluation of Winter Ticks (Dermacentor albipictus) Collected from North American Elk (Cervus canadensis) in an Area of Chronic Wasting Disease Endemicity for Evidence of PrPCWD Amplification Using Real-Time Quaking-Induced Conversion Assay. mSphere 6, e0051521 (2021).

43. M. Rossi, et al., Ultrasensitive RT-QuIC assay with high sensitivity and specificity for Lewy bodyassociated synucleinopathies. Acta Neuropathol. 140, 49-62 (2020).

44. T. H. Mok, et al., Bank vole prion protein extends the use of RT-QuIC assays to detect prions in a range of inherited prion diseases. Sci. Rep. 11, 5231 (2021).

45. A. Wang, Y. R. Perera, M. B. Davidson, N. C. Fitzkee, Electrostatic Interactions and Protein Competition Reveal a Dynamic Surface in Gold Nanoparticle-Protein Adsorption. J. Phys. Chem. C 120, 24231-24239 (2016).

46. Anika Gladytz Bernd Abel, H. J. Risselada, Gold-induced fibril growth: the mechanism of surfacefacilitated amyloid aggregation.pdf. Angew. Chem. Int. Ed. 55, 11242-11246 (2016).

47. E. Gasteiger, et al., Protein Identification and Analysis Tools on the ExPASy Server. The Proteomics Protocols Handbook, 571-607 (2005).

48. E. Csapó, et al., Surface and Structural Properties of Gold Nanoparticles and Their Biofunctionalized Derivatives in Aqueous Electrolytes Solution. J. Dispers. Sci. Technol. 35, 815-825 (2014).

49. X. Zhang, Gold Nanoparticles: Recent Advances in the Biomedical Applications. Cell Biochem. Biophys. 72, 771-775 (2015).

50. J. Li, X. Yan, X. Li, X. Zhang, J. Chen, A new electrochemical immunosensor for sensitive detection of prion based on Prussian blue analogue. Talanta 179, 726-733 (2018). 
51. J. Zhao, et al., Graphene oxide-gold nanoparticle-aptamer complexed probe for detecting amyloid beta oligomer by ELISA-based immunoassay. J. Immunol. Methods 489, 112942 (2021).

52. K. Mahato, et al., Gold nanoparticle surface engineering strategies and their applications in biomedicine and diagnostics. 3 Biotech 9, 57 (2019).

53. J.-H. Lee, H.-Y. Cho, H. K. Choi, J.-Y. Lee, J.-W. Choi, Application of Gold Nanoparticle to Plasmonic Biosensors. Int. J. Mol. Sci. 19 (2018).

54. R. Adato, et al., Ultra-sensitive vibrational spectroscopy of protein monolayers with plasmonic nanoantenna arrays. Proc. Natl. Acad. Sci. U. S. A. 106, 19227-19232 (2009).

55. M. A. Wild, T. R. Spraker, C. J. Sigurdson, K. I. O'Rourke, M. W. Miller, Preclinical diagnosis of chronic wasting disease in captive mule deer (Odocoileus hemionus) and white-tailed deer (Odocoileus virginianus) using tonsillar biopsy. J. Gen. Virol. 83, 2629-2634 (2002).

56. N. C. Ferreira, et al., Detection of chronic wasting disease in mule and white-tailed deer by RTQuIC analysis of outer ear. Sci. Rep. 11, 7702 (2021).

57. D. Gao, et al., Studies on the interaction of colloidal gold and serum albumins by spectral methods. Spectrochim. Acta A Mol. Biomol. Spectrosc. 62, 1203-1208 (2005).

58. G. P. Szekeres, J. Kneipp, Different binding sites of serum albumins in the protein corona of gold nanoparticles. Analyst 143, 6061-6068 (2018).

59. A. Kraus, et al., Structure of an infectious mammalian prion. bioRxiv, 2021.02.14.431014 (2021).

60. K. A. Matthews, et al., Racial and ethnic estimates of Alzheimer's disease and related dementias in the United States (2015-2060) in adults aged $\geq 65$ years. Alzheimers. Dement. 15, 17-24 (2019).

61. S. Hwang, J. J. Greenlee, E. M. Nicholson, Use of bovine recombinant prion protein and real-time quaking-induced conversion to detect cattle transmissible mink encephalopathy prions and discriminate classical and atypical L- and H-Type bovine spongiform encephalopathy. PLoS One 12, e0172391 (2017).

62. G. Fairfoul, et al., Alpha-synuclein RT-QuIC in the CSF of patients with alpha-synucleinopathies. Ann Clin Transl Neurol 3, 812-818 (2016).

63. N. Salvadores, M. Shahnawaz, E. Scarpini, F. Tagliavini, C. Soto, Detection of misfolded A $\beta$ oligomers for sensitive biochemical diagnosis of Alzheimer's disease. Cell Rep. 7, 261-268 (2014).

64. T. Špringer, J. Homola, Biofunctionalized gold nanoparticles for SPR-biosensor-based detection of CEA in blood plasma. Anal. Bioanal. Chem. 404, 2869-2875 (2012). 